\title{
Infections of cardiac implantable electronic devices: Epidemiology, classification, treatment, and prognosis
}

\author{
Grzegorz Sławiński ${ }^{A-F}$, Ewa Lewicka ${ }^{A-F}$, Maciej Kempa ${ }^{A-F}$, Szymon Budrejko ${ }^{A-F}$, Grzegorz Raczak ${ }^{A-F}$ \\ Department of Cardiology and Electrotherapy, Medical University of Gdańsk, Poland \\ A - research concept and design; $\mathrm{B}$ - collection and/or assembly of data; $\mathrm{C}$ - data analysis and interpretation; \\ $D$ - writing the article; $E$ - critical revision of the article; $F$ - final approval of the article
}

Address for correspondence

Maciej Kempa

E-mail: kempa@gumed.edu.pl

Funding sources

None declared

Conflict of interest

None declared

Received on February 19, 2017

Reviewed on April 20, 2017

Accepted on November 20, 2017

Published online on July 26, 2018

Cite as

Sławiński G, Lewicka E, Kempa M, Budrejko S, Raczak G.

Infections of cardiac implantable electronic devices: Epidemiology, classification, treatment, and prognosis. Adv Clin Exp Med. 2019;28(2):263-270. doi:10.17219/acem/80665

D0I

10.17219/acem/80665

Copyright

Copyright by Author(s)

This is an article distributed under the terms of the

Creative Commons Attribution Non-Commercial License

(http://creativecommons.org/licenses/by-nc-nd/4.0/)

\begin{abstract}
The increasing number of implantation procedures of implantable cardiac electronic devices (ICEDs) leads to a substantial growth of a cohort of patients in whom complications of such a therapy occur. Infective complications are among the most severe ones, as they are often associated with poor prognosis. Depending on the criteria applied, the incidence of cardiac device infection (CDI) is estimated at $0.5-2.2 \%$. Many risk factors of CDIs have been identified, among which the most important are numerous previous cardiac electrotherapy procedures and their complexity, and the lack of perioperative antibiotic prophylaxis. Appropriate diagnosis of a suspected CDI is of utmost importance, as well as the correct classification of the infection, which leads to adequate treatment. Management of a CDI should include complete removal of the implanted device. Additionally, empirical and then targeted antibiotic therapy should be instituted. The prognosis of CDI may, nonetheless, be unfavorable. Despite appropriate treatment, the total mortality rate of such complication is estimated to be as high as 35\%.
\end{abstract}

Key words: infective endocarditis, implantable cardioverter-defibrillator, implantable cardiac electronic device, cardiac pacemaker, infective complications 


\section{Introduction}

Cardiac device infections (CDIs) of cardiac pacemakers, implantable cardioverters-defibrillators (ICDs) or resynchronization therapy devices (Cardiac Resynchronization Therapy Pacemaker or Cardiac Resynchronization Therapy Defibrillator: CRT-P or CRT-D) belong to the most significant clinical problems of contemporary cardiology. The increasing number of implantation procedures of implantable cardiac electronic devices (ICEDs) leads to a substantial growth of a cohort of patients in whom complications of such a therapy occur. Infective complications are among the most severe ones, as they are often associated with poor prognosis. CDIs create various clinical problems, including diagnosis, classification of infections and treatment.

\section{Epidemiology}

Depending on the criteria applied, the incidence of CDI is estimated at $0.5-2.2 \% .^{1}$ Available research data shows that the infection rate increases along with the increasing complexity of the implantable device system; infection is least frequent in pacemaker patients, more frequent in patients with an ICD and most frequent in biventricular resynchronization therapy patients (with CRT-P or CRT-D devices). Most studies point to such a relationship, except one with contrary conclusions, which - according to the authors' opinion - may be due to the limited number of patients in the cohort with CRT systems. ${ }^{2}$ Higher frequency of CDI in patients with more complex systems may be the consequence of longer procedural times in those patients, which was confirmed in some of the available studies. ${ }^{3,4}$

The incidence of CDIs is from 2-fold to 5 -fold lower in the case of first-time procedures, as compared to subsequent revisions of the implanted system. It is estimated that infections complicate $0.5-0.8 \%$ of first-time implantations and $1-4 \%$ of system revisions. ${ }^{5-7}$

\section{Risk factors}

Cardiac device infections constitute a serious medical problem, but they also influence the economic balance of healthcare systems. Therefore, numerous studies have been undertaken to determine the risk factors of that complication. Among the most important factors mentioned is the number of previous cardiac electrotherapy procedures performed in a single patient, ${ }^{2,5,8,9}$ the complexity of those procedures $3,6,10,11$ and the lack of perioperative antibiotic prophylaxis. ${ }^{4,5,8,11}$ Other important risk factors of infective complications (confirmed in at least 2 studies) comprise the following: male sex, ${ }^{8,12}$ younger age, ${ }^{8,10}$ anticoagulation, ${ }^{2,12}$ chronic obstructive pulmonary disease, ${ }^{9,13}$ chronic kidney disease, ${ }^{2,12}$ and reoperation during the same hospitalization. ${ }^{3,7}$ Additional CDI risk factors, though less documented, include: procedure performed before the year $1985,{ }^{8}$ fever during $24 \mathrm{~h}$ preceding the procedure, ${ }^{5}$ temporary cardiac pacing before the procedure, ${ }^{5}$ concomitant chronic heart failure, ${ }^{12}$ chronic systemic glucocorticosteroid therapy, ${ }^{11}$ hemodialysis, ${ }^{3}$ prolonged duration of the procedure, ${ }^{3}$ and pocket hematoma. ${ }^{4}$

Sohail et al. determined the risk factors of early infection after ICD implantation. ${ }^{13}$ They showed that the risk of early ICD system infection is substantially increased by the presence of epicardial leads and perioperative ICD pocket complications - pocket hematoma, wound dehiscence and delayed wound healing.

The total mortality in the case of CDIs is estimated at 0-35\%. Those numbers come from 19 studies, comprising at least 1,000 patients, with the follow-up period of up to 5.5 years. ${ }^{11,14-26}$ The reported mortality in the course of CDIs increased along with the prolongation of the follow-up period. It was $2-15 \%$ during the first 30 days after implantation procedure, ${ }^{14,21,24,27,28} 4-29 \%$ during 6 months, ${ }^{14,19,21,26} 9-35 \%$ during the first year after implantation, ${ }^{16,22-24,28}$ and $6-35 \%$ if at least 2 years of follow-up were taken into account. ${ }^{15,17,20,22,29}$ It is of note that the data cited above included heterogeneous cohorts of patients with various manifestations of CDIs. Higher mortality was reported in studies where authors qualified only patients with a systemic form of CDI - 24.5-29\% during 1-year follow-up, ${ }^{14,21,24}$ as compared to studies reporting mortality in patients with only a local manifestation of the infection - 6\% during 2-year follow-up. ${ }^{17}$ Also, in studies of patient cohorts with any form of CDI, the reported mortality during 1-year follow-up was higher in systemic forms of CDI - 15.5-26\%, as compared to local manifestations of CDI $-1.5-12.5 \% .^{20,29}$

Because of such an unfavorable course of CDI, many research teams have investigated risk factors of poor prognosis in patients with ICEDs. Among those, renal insufficiency has been most frequently reported. ${ }^{20,26,30}$ Other risk factors of poor prognosis include the diagnosis of infective endocarditis (as well as peripheral thromboembolism or moderate/severe tricuspid regurgitation, indicating infective endocarditis) ${ }^{26,30}$ and patients' older age. ${ }^{20,30}$ Moreover, patients treated conservatively had a higher mortality rate than those in whom the complete removal of the previously implanted system was performed. ${ }^{22,24,30}$

\section{Classification}

In recent years, several classifications have been proposed to describe patients with CDIs. At present, it seems that the classification given by Sandoe et al. may be very useful in everyday clinical practice, and its use is advised also in Poland..$^{1}$ The authors propose to distinguish 4 basic clinical manifestations of CDIs, such as uncomplicated infection of the pulse generator, complicated infection of the 
pulse generator, lead infection, and infective endocarditis in a patient with an ICED (Fig. 1).

In uncomplicated pulse generator infection, no signs or symptoms of a systemic infection may be found, and blood cultures are negative. Uncomplicated generator pocket infection (PI) may be diagnosed in the case of (Fig. 2):

- spreading cellulitis of the pocket;

- purulent exudate from the incision site;

- wound dehiscence or erosion through skin with the exposure of the generator or leads;

- pocket fistula or abscess formation.

Complicated pulse generator infection is diagnosed if the abovementioned PI signs are met, but with evidence of lead or endocardial involvement, systemic signs or symptoms of infection, or positive blood cultures.

Definite lead-related infective endocarditis (LRIE) is diagnosed in the case of:

- symptoms or signs of systemic infection, presence of vegetations attached to leads, as determined by echocardiography, and presence of major Duke microbiological criteria, with no signs of pulse generator PI; or

- symptoms or signs of systemic infection, and microbiological culture, histological or molecular evidence of infection of explanted lead, with no signs of pulse generator PI.

Possible LRIE may be diagnosed in the case of:

- symptoms or signs of systemic infection, presence of vegetations attached to leads, as determined by echocardiography, when major Duke microbiological criteria are not met; or

- symptoms or signs of systemic infection, presence of major Duke microbiological criteria, with no echocardiographic evidence of vegetations attached to leads.

Implantable cardiac electronic device-associated infective endocarditis (ICED-IE) may be diagnosed, if Duke criteria for infective endocarditis are met and infection

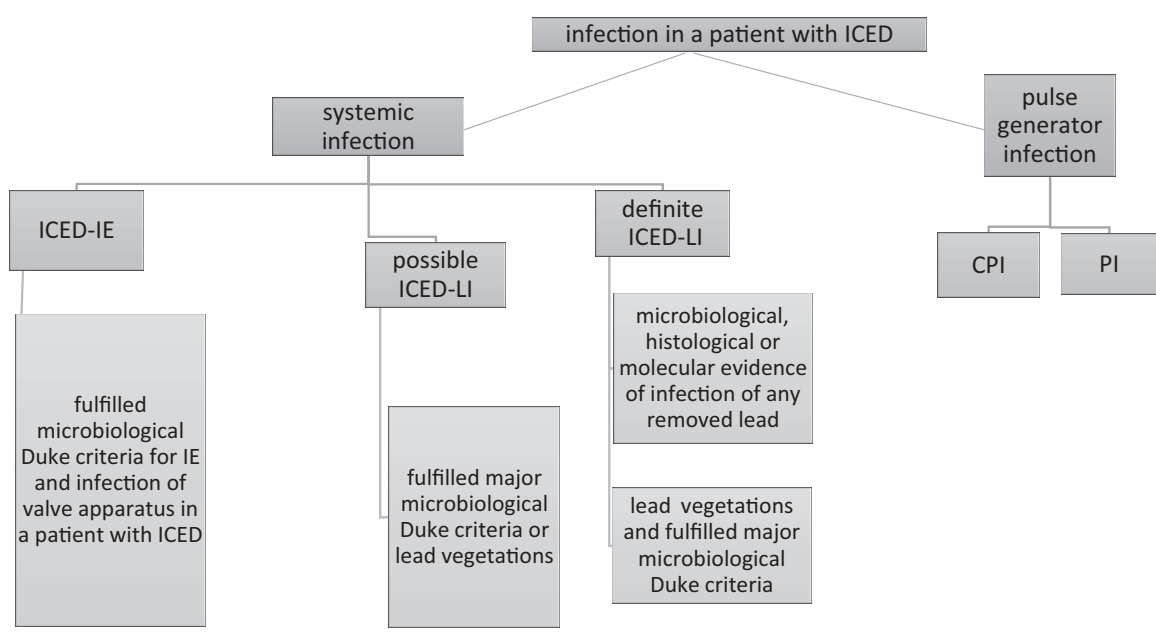

Fig. 1. Classification of implantable cardiac electronic device infections

$\mathrm{PI}$ - pocket infection; CPI - complicated pocket infection; ICED - implantable cardiac electronic device; ICED-IE - implantable cardiac electronic device-associated infective endocarditis;

ICED-LI - implantable cardiac electronic device isolated lead infection.

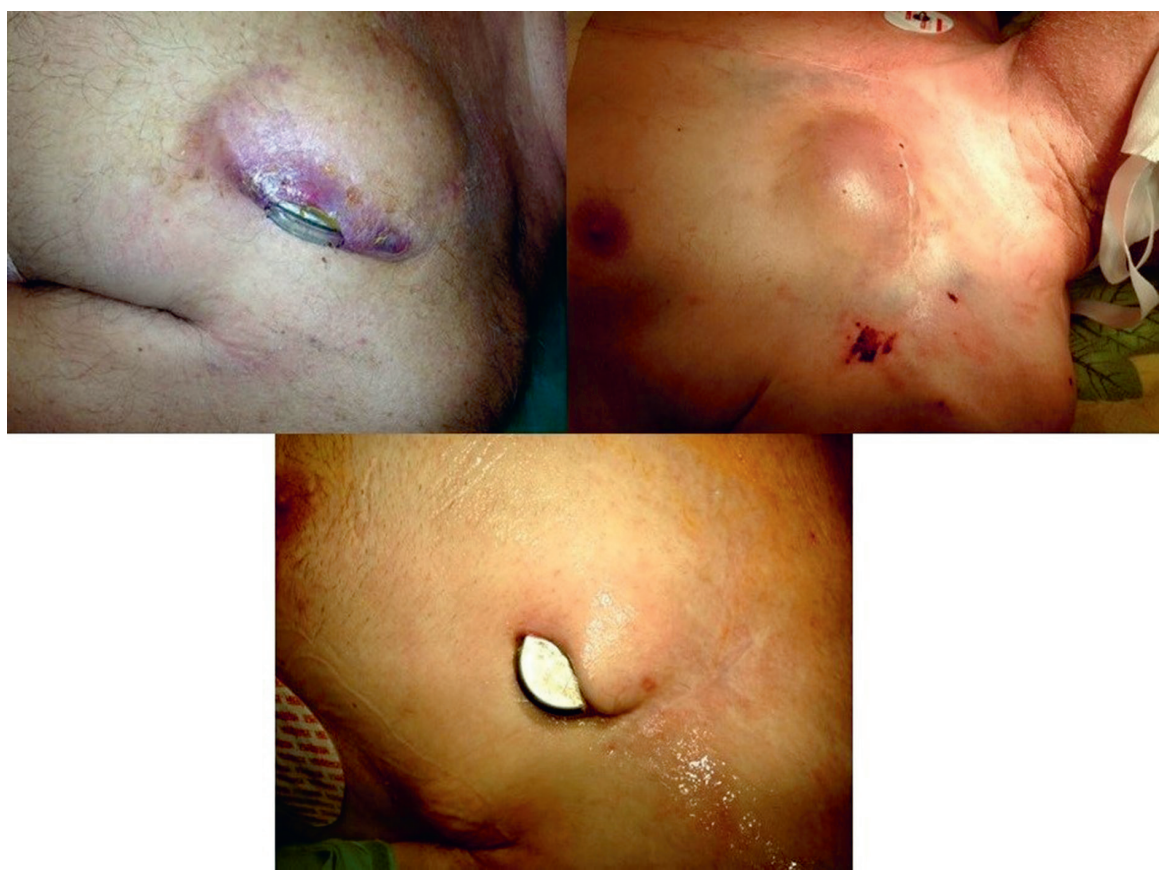

Fig. 2. Examples of clinical manifestations of pocket infection (from Authors' archive) 
of the valve apparatus is confirmed with echocardiography (Fig. 1).

Apart from the above definitions, it is proposed to include the following as the major diagnostic criteria of ICED isolated lead infection (ICED-LI) and ICED-IE:

- infection of the pulse generator pocket;

- vegetations revealed with fluorine-18-fluorodeoxyglucose positron emission tomography/computed tomography (18F-FDG-PET/CT) or radio-labeled leukocyte single-photon emission computed tomography combined with computed tomography (SPECT/CT), or vegetations in the pulmonary circulation demonstrated with angio-computed tomography (CT);

- recurrent pneumonia in a patient with an ICED (as a manifestation of septic pulmonary embolism).

\section{Etiology}

The main etiological factor of CDIs are Gram-positive bacteria, which are responsible for $67.2-92.5 \%$ of the total number of infections, while Gram-negative bacteria account for less than $18 \%$ of infections. Among Gram-positive bacteria, coagulase negative staphylococci (10-68\% of infections) and Staphylococcus aureus (24-59\%) 14,19,20,23,24,31-34 are most often involved in CDIs. Bacteria that are less frequently involved in CDIs are Gram-negative rods (1-17\% of infections), enterococci (5-6\%), streptococci $(4-6 \%)$, propionibacteria and fungi $(0.5-2 \%)$. Moreover, it is estimated that in $2-24.5 \%$ of cases, the CDI is caused by several species. ${ }^{15-19,31,33}$ What is of importance is that in $12-49 \%$ of patients with clinical manifestations of CDI, blood cultures are negative. ${ }^{7,14,15,19-21,24,26,31,33-35}$

\section{Clinical manifestation}

Signs and symptoms of CDI depend on the clinical type of infection. In the case of isolated PI, they include redness, regional edema and tenderness of the pocket region. In more advanced types of infection, purulent exudate may appear, as well as wound dehiscence, fistula formation or erosion of the subcutaneous tissue over the pocket. ${ }^{36}$ Spreading infection may affect the leads of the implanted system. Literature data shows that PI coexists with ICED-IE and ICED-LI in 6-58\% of cases, depending on the methodology applied and the classification of infections. ${ }^{7,15,26,37,38}$

The diagnosis of ICED-LI or ICED-IE in patients in whom no signs of PI are present poses a great challenge. Patients with ICED-IE or ICED-LI often present non-specific signs and symptoms of systemic infection, such as fever, shivering, nocturnal sweat, and weakness. Laboratory tests show elevated C-reactive protein (CRP) levels, which are observed in up to $96 \%$ of patients, but it is not useful to differentiate PI, ICED-LI and ICED-IE. ${ }^{24,36,39,40}$ In $9 \%$ of patients with ICED-LI and ICED-IE, signs of septic shock may occur, and $5 \%$ of patients present with symptoms of vascular involvement or pulmonary embolism. ${ }^{41}$ Patients with ICED-LI and ICED-IE quite often report dyspnea and chest pain of pleural characteristics, with radiological features of pulmonary tissue involvement. Secondary infective foci, manifesting as vertebral body infection or intervertebral disc infection, ${ }^{40}$ are additional signs of possible ICED-IE or ICED-LI.

\section{Diagnostics}

Patients with suspected CDI may require numerous imaging procedures before the final diagnosis is established. According to current guidelines, in every patient with suspected CDI, a chest X-ray is required to determine the pulse generator location, the number of leads and their location, the possible presence of a pulmonary inflammatory process, and pleural effusion.

Echocardiography should be performed as soon as possible in every patient with suspected CDI (the optimal time range is within the first $24 \mathrm{~h}$ of the diagnostic work-up). It has been shown that early system removal (within the first 3 days from the diagnosis) is associated with a significantly better prognosis. ${ }^{42}$ Echocardiography is also essential in patients with PI due to the frequent coexistence with ICED-LI or ICED-IE. The purpose of echocardiographic examination is to search for possible endocardial or leadassociated vegetations, new valvular insufficiency or abscess formation. In the case of suspected ICED-LI or ICEDIE, transesophageal echocardiography (TEE) is indicated, as its sensitivity to reveal the abovementioned abnormalities is much higher, compared to transthoracic echocardiography (TTE). Observational studies have shown that TTE enables the detection of lead involvement in 22-43\% of cases and TEE in $90-96 \%$ of cases. ${ }^{21,39-41}$ However, one must remember that both methods are complementary. Transthoracic echocardiography enables a more precise evaluation of the left ventricle, the size of right heart chambers and right ventricular pressure, whereas TEE enables a more precise assessment of the intracardiac and extracardiac segments of implanted leads, left heart valvular involvement, and the number, size and mobility of vegetations. It is advised to repeat the echocardiography once the ICED has been removed to exclude the presence of remaining vegetations within the valvular apparatus.

It has been shown that FDG PET/CT may be a valuable imaging modality that is helpful in the diagnostic process of CDIs. ${ }^{43}$ However, because of lacking evidence, it is not recommended as a clinical routine, but only as a complementary test in the case of infections of an atypical clinical course.

Diagnostics of suspected CDI comprises an appropriate number of blood cultures, sampled in proper time periods. It is vital to collect blood samples before antibiotic therapy 
is instituted. According to the British recommendations, the number of blood samples depends on the clinical status of a patient with suspected CDI. ${ }^{1}$ In a patient that is clinically stable, in whom subacute or chronic infection is suspected, it is recommended to collect 3 blood culture samples separated by at least 6 -hour intervals. In a patient with CDI and severe sepsis or septical shock, to avoid any delay in empirical antibiotic therapy, it is recommended to collect 2 blood culture samples separated by 1-hour time interval before antibiotic therapy is started. Following the above recommendations allows obtaining positive blood cultures in 20-67\% of patients with CDIs. 4,10,31,44

Apart from blood cultures, it is recommended to perform a microbiological investigation of the removed lead or its fragments (optimally, both proximal and distal segments), vegetations collected from leads and pocket tissues, or purulent exudate from the pocket, if present.

Available data shows low accordance of blood culture results and cultures of removed lead fragments (35\%). This should not be surprising, as blood cultures are collected before antibiotic therapy is started, and leads are removed and referred for a microbiological investigation after some period of antibiotic pharmacotherapy. ${ }^{31}$

\section{Management}

Management of any diagnosed infection (PI, ICED-LI or ICED-IE) comprises several common points. In each of the infection types, it is advised to remove totally, if possible, the infected system (i.e., the pulse generator and leads). The procedure should be performed as soon as possible and not later than 2 weeks from the diagnosis. Patients should receive antibiotics according to antibiograms obtained from blood cultures. Still, even if an appropriate management strategy is applied, CDI recurs in up to $7 \%$ of patients. ${ }^{11,14-19}$ What is of note, infection recurrence is more likely if an ICED system is left in place. It is estimated that over a half of patients that has not undergone ICED system removal will develop the next CDI episode..$^{10,17,45}$

Also, in the cases of pocket decubitus or the exposition of leads to extracorporeal environment, as bacterial contamination is inevitable, complete removal of the implanted system is recommended. If local and systemic signs and symptoms of infection are excluded, which means a diagnosis of the so-called "sterile decubitus", prolonged antibiotic treatment is not required, and only perioperative prophylaxis is applied. ${ }^{1}$

Transvenous lead extraction and pulse generator removal (Fig. 3) may be successfully performed in $98 \%$ of patients. ${ }^{46}$ The risk of failure of such a procedure increases along with the prolongation of lead dwelling time in the venous system. In one of the studies, the authors found a linear correlation between the system dwelling time (from the initial implantation to the removal procedure) and the risk of the removal failure. In the case of leads implanted up to 3 years before, the risk of failure was $5 \%$, and for the leads implanted from 9 to 12 years before, it was as high as $20 \% .{ }^{38}$ Cardiac surgical removal of an ICED is recommended in patients with large vegetations $(>2 \mathrm{~cm}$ in diameter) and in those requiring valve replacement or repair because of infective endocarditis.

Complete removal of the implanted system may not always be possible. It may happen in the case of procedural failure, when some parts of leads remain in the patient's circulation, but also if a patient refuses the ICED removal ${ }^{17}$ or if a patient is disqualified from the procedure because of poor prognosis due to severe concomitant diseases. ${ }^{5,7}$ That may be the case in $3-15 \%$ of patients with CDI. ${ }^{4,5,7,10,37,47}$ Management of such cases includes typical antibiotic therapy with leads left in situ, or alternatively the removal of the pulse generator in the case of confirmed PI. It has been shown that partial removal of an ICED system leads to infection cure in $13-71 \%$ of patients. ${ }^{10,16,17,37,47}$

If ICED extraction is planned, it is obligatory to reassess current indications for cardiac electrotherapy. It is estimated that up to $30 \%$ of patients do not require repeat implantation of the removed ICED. ${ }^{47}$ In other cases, the repeat implantation procedure should be delayed (if possible) until general (fever) and local symptoms of infection have passed. Some authors suggest to maintain an arbitrary delay of 7-10 days from the system extraction to reimplantation. ${ }^{7,37}$ The use of ipsilateral vascular access is discouraged. No part of the previously removed system is allowed to be reimplanted.

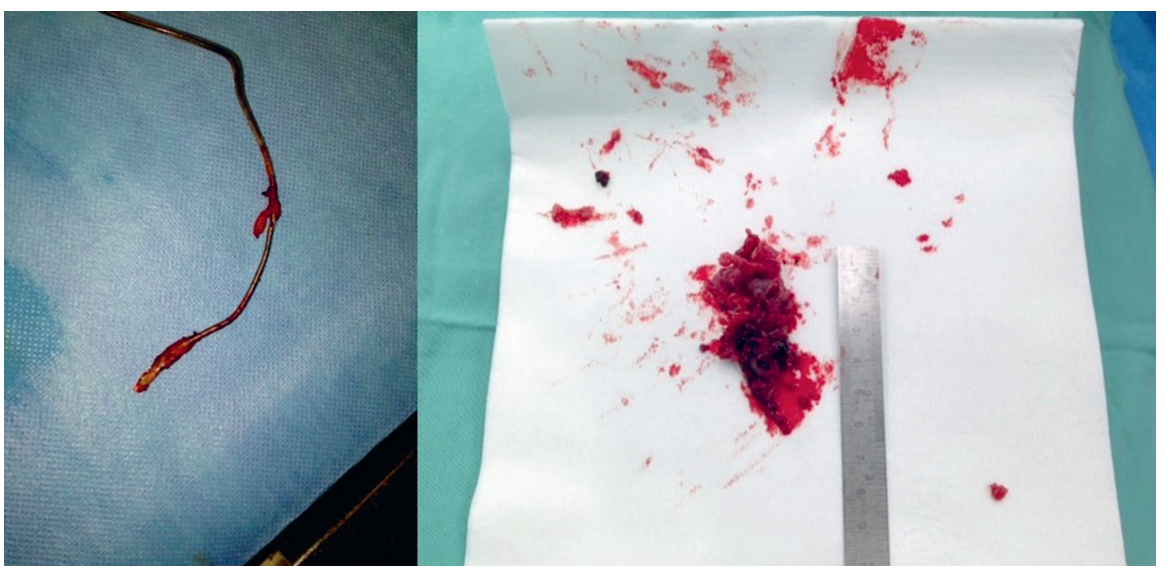

Fig. 3. The left panel pictures a pacing lead that was removed transvenously because of infection (ICED-LI); the right panel pictures vegetations removed during cardiac surgery (from Authors' archive)

ICED-LI - implantable cardiac electronic device isolated lead infection. 
In exceptional situations, in the case of infection limited to the superficial tissue or skin incision only, after detailed exclusion of the infective involvement of the pocket, implanted leads and bacterial contamination of the abovementioned structures, it is acceptable to apply conservative treatment, which means 7-10 days of oral antibiotic treatment (with an antistaphylococcal agent) with possible local surgical debridement. Nevertheless, in those exceptionally rare cases, the region of inflammatory involvement should be thoroughly inspected and monitored, and in the case of progression of the infection into deeper tissue layers, a typical treatment as for CDI should be instituted immediately, with complete removal of the implanted system. ${ }^{48}$

\section{Antimicrobial therapy}

Appropriate antimicrobial therapy is an indispensable part of the management strategy in patients with CDIs. The type of antibiotics used and the duration of treatment depend on the extent of infection and the clinical state of a patient. In the case of uncomplicated PI, empirical antibiotic therapy is necessary until the results of collected cultures may be obtained. Successful empirical antibiotic therapy usually includes vancomycin, daptomycin or teicoplanin. Local antibiotic treatment is not recommended. In the case of complicated PI, a typical treatment as for ICED-LI or ICED-IE should be implemented.

Obviously, the diagnosis of ICED-LI or ICED-IE also requires the initiation of appropriate empirical antibiotic therapy. Until blood culture results are available, and if the patient deteriorates (e.g., in severe sepsis), it is advised to use vancomycin with meropenem or daptomycin with meropenem. In patients with ICED-IE or ICED-LI with negative blood cultures, it is recommended to use vancomycin with gentamycin or daptomycin with gentamycin.

Empirical antibiotic therapy should include broad-spectrum agents; however, the efficacy of such a strategy was lower when compared to antibiogram-guided therapy. For example, flucloxacillin is far more effective as a therapy of Staphylococcus aureus infections than vancomycin. No randomized clinical trials have been conducted to assess the efficacy of each therapy regimen in the case of ICED-IE or ICED-LI. Moreover, many publications lack detailed information about the course of antibiotic treatment, such as the dosage, route of administration, duration of treatment, or the species of the pathogen responsible for infection. 5,7,8,37,44

For the antimicrobial treatment of CDI, peripheral venous access is recommended. It is characterized by a lower risk of secondary infection and it does not result in the loss of a potential site for future reimplantation. Peripheral venous access is thus the best solution in the case of prolonged antibiotic administration, but it should be changed on a regular basis every $72 \mathrm{~h} .{ }^{25}$ Oral route of antibiotic administration is not recommended in CDIs. It was shown that oral antibiotics for PI not only did not cure the infection, but also increased the risk of infection dissemination and mortality. ${ }^{27}$ Oral antibiotic therapy is allowed only in the case of persistent cellulitis after system extraction and in patients with no chance for ICED system extraction (subjects in the terminal phase of a chronic disease).

The duration of antibiotic treatment depends on several factors: type of CDI, management of the infected system, involvement of other cardiac structures, and extracardiac signs of infection. In the case of PI, experts' opinions are consistent and the recommended duration of antibiotic therapy is 10-14 days. ${ }^{4,20,44}$

In the case of ICED-LI or ICED-IE, antibiotic therapy is administered for 6 weeks ${ }^{15,20,21,45}$; however, an average of 5.4 weeks ${ }^{35}$ and $2-4$ weeks were also reported. ${ }^{47}$ In patients with ICED-LI or ICED-IE with no ICED system extraction, the British recommendation is 6 weeks of antibiotic therapy. ${ }^{1}$ It is generally recommended to stop the therapy after 6 weeks, with the following careful reassessment of the patient's clinical status, and to repeat blood cultures in the case of any suspicion of infection recurrence. There are no strict recommendations regarding the management of infection recurrences. It is advised to start with intravenous antibiotic therapy and subsequent long-lasting oral therapy. ${ }^{7}$

The duration of antibiotic treatment after the extraction of an infected ICED system is determined by several factors, such as the presence of extracardiac infective foci and the initial clinical response to treatment; it also depends on whether the valvular apparatus is involved. With good clinical response and no extracardiac foci, it seems sufficient to continue antibiotic therapy for 4 weeks after the extraction procedure. If CDI occurred in a patient with a mechanical valve prosthesis, a longer 6 -week period is recommended. It should be underlined that every change of the treatment scheme (e.g., in case of inefficacy of the previous plan) should reset the time counter again for the complete period, as described above.

\section{Prevention}

Performing ICED system implantation or revision only by experienced electrophysiologists in appropriately equipped centers is very important in terms of CDIs prevention. Temporary transvenous cardiac pacing before ICED implantation is a risk factor of CDI. It is associated with $2-18 \%$ risk of sepsis ${ }^{49}$ and a 2.5 -fold increase of the risk of future CDI. ${ }^{5}$

Elective implantation procedures should be postponed until the complete regression of general symptoms (mainly fever) of any infection. It would be interesting to investigate the prognostic value of CRP concentration and white blood cells count before the implantation procedure for the prediction of future CDI.

Based on a meta-analysis of results from randomized clinical trials, it has been established that prophylactic antibiotic administration before the implantation procedure 
is an effective method of prevention of CDI. ${ }^{50,51}$ Cefazolin has best evidence as an effective antibiotic, preventing CDI. ${ }^{4}$ Flucloxacillin compared to placebo did not show any clinical benefit. ${ }^{52}$ It is recommended to administer the antibiotic before the implantation procedure, $1 \mathrm{~h}$ before skin incision. ${ }^{50}$ This antibiotic scheme allows one to achieve tissue and plasma antibiotic concentration above minimal inhibitory concentration (MIC) for potential pathogens. It is not recommended to repeat the antibiotic after the procedure. ${ }^{50,53}$

A crucial part of CDI prevention is appropriate skin preparation in the region of the planned skin incision. Hair should be regionally removed with the use of electric clippers with a single-use head, because the use of razors is associated with a higher risk for infection of the tissue operated on. Hair should be removed on the day of the planned procedure. Recommended antiseptic agents include alcoholic $2 \%$ chlorhexidine solution or povidone iodine in alcohol, but notably the use of alcoholic $2 \%$ chlorhexidine is associated with higher antiseptic efficacy as compared to povidone iodine in alcohol. ${ }^{54,55}$ Therefore, British recommendations postulate the use of alcoholic $2 \%$ chlorhexidine solution as the agent of choice for antiseptic preoperative skin preparation, and obeying the crucial rule to leave it on the skin until it dries, which usually takes approx. $30 \mathrm{~s}^{1}$

\section{Summary}

CDIs still constitute a serious clinical problem. It is of particular difficulty to establish the final diagnosis in patients with a deceitful and chronic course of the disease. New imaging techniques, such as 18F-FDG-PET/CT or radio-labeled leukocyte SPECT/CT, show high sensitivity for CDI detection. Future studies are needed to assess whether laboratory tests (CRP, procalcitonin, leukocytosis) are useful in predicting CDI occurrence. With increasing resistance of pathogens to antibiotics, further studies are required to compare the efficacy of various therapeutic algorithms, as well as the search for new antimicrobial drugs.

\section{References}

1. Sandoe JA, Barlow G, Chambers JB, et al. Guidelines for the diagnosis, prevention and management of implantable cardiac electronic device infection. Report of a joint Working Party project on behalf of the British Society for Antimicrobial Chemotherapy (BSAC, host organization), British Heart Rhythm Society (BHRS), British Cardiovascular Society (BCS), British Heart Valve Society (BHVS) and British Society for Echocardiography (BSE). J Antimicrob Chemother. 2015;70: 325-359.

2. Lekkerkerker JC, van Nieuwkoop C, Trines SA, et al. Heart rhythm disorders and pacemakers: Risk factors and time delay associated with cardiac device infections: Leiden device registry. Heart. 2009;95: 715-720.

3. Romeyer-Bouchard C, Da Costa A, Dauphinot V, et al. Prevalence and risk factors related to infections of cardiac resynchronization therapy devices. Eur Heart J. 2010;31:203-210.
4. de Oliveira JC, Martinelli M, Nishioka SA, et al. Efficacy of antibiotic prophylaxis before the implantation of pacemakers and cardioverter-defibrillators: Results of a large, prospective, randomized, doubleblinded, placebo-controlled trial. Circ Arrhythm Electrophysiol. 2009; 2:29-34.

5. Klug D, Balde M, Pavin D, et al. Risk factors related to infections of implanted pacemakers and cardioverter-defibrillators: Results of a large prospective study. Circulation. 2007;116:1349-1355.

6. Nery PB, Fernandes R, Nair GM, et al. Device-related infection among patients with pacemakers and implantable defibrillators: Incidence, risk factors, and consequences. J Cardiovasc Electrophysiol. 2010;21:786-790.

7. Catanchin A, Murdock CJ, Athan E. Pacemaker infections: A 10-year experience. Heart Lung Circ. 2007;16:434-439.

8. Johansen JB, Jorgensen $O D$, Moller $M$, et al. Infection after pacemaker implantation: Infection rates and risk factors associated with infection in a population-based cohort study of 46,299 consecutive patients. Eur Heart J. 2011;32:991-998.

9. Landolina M, Gasparini M, Lunati M, et al. Long-term complications related to biventricular defibrillator implantation: Rate of surgical revisions and impact on survival: Insights from the Italian Clinical Service Database. Circulation. 2011;123:2526-2535.

10. Margey R, McCann H, Blake G, et al. Contemporary management of and outcomes from cardiac device related infections. Europace. 2010;12:64-70.

11. Sohail MR, Uslan DZ, Khan AH, et al. Risk factor analysis of permanent pacemaker infection. Clin Infect Dis. 2007;45:166-173.

12. Bloom $\mathrm{H}$, Heeke $B$, Leon $A$, et al. Renal insufficiency and the risk of infection from pacemaker or defibrillator surgery. Pacing Clin Electrophysiol 2006;29:142-145.

13. Sohail MR, Hussain S, Le KY, et al. Risk factors associated with earlyversus late-onset implantable cardioverter-defibrillator infections. J Interv Card Electrophysiol. 2011;31:171-183.

14. Grammes JA, Schulze CM, Al-Bataineh M, et al. Percutaneous pacemaker and implantable cardioverter-defibrillator lead extraction in 100 patients with intracardiac vegetations defined by transesophageal echocardiogram. J Am Coll Cardiol. 2010;55:886-894.

15. Knigina L, Kühn C, Kutschka I, et al. Treatment of patients with recurrent or persistent infection of cardiac implantable electronic devices. Europace. 2010;12:1275-1281.

16. Chua JD, Wilkoff BL, Lee I, et al. Diagnosis and management of infections involving implantable electrophysiologic cardiac devices. Ann Intern Med. 2000;133:604-608.

17. Klug D, Wallet F, Lacroix D, et al. Local symptoms at the site of pacemaker implantation indicate latent systemic infection. Heart. 2004;90: 882-886.

18. Tascini C, Bongiorni MG, Gemignani G, et al. Management of cardiac device infections: A retrospective survey of a non-surgical approach combining antibiotic therapy with transvenous removal. J Chemother. 2006;18:157-163.

19. Viola GM, Awan LL, Darouiche RO. Nonstaphylococcal infections of cardiac implantable electronic devices. Circulation. 2010;121: 2085-2091.

20. Deharo JC, Quatre A, Mancini J, et al. Long-term outcomes following infection of cardiac implantable electronic devices: A prospective matched cohort study. Heart 2012;98:724-731.

21. Greenspon AJ, Prutkin JM, Sohail MR, et al. Timing of the most recent device procedure influences the clinical outcome of lead-associated endocarditis results of the MEDIC (Multicenter Electrophysiologic Device Infection Cohort). J Am Coll Cardiol. 2012;59:681-687.

22. Le KY, Sohail MR, Friedman PA, et al. Impact of timing of device removal on mortality in patients with cardiovascular implantable electronic device infections. Heart Rhythm. 2011;8:1678-1685.

23. Tarakji KG, Chan EJ, Cantillon DJ, et al. Cardiac implantable electronic device infections: Presentation, management, and patient outcomes. Heart Rhythm. 2010;7:1043-1047.

24. Athan E, Chu VH, Tattevin P, et al. Clinical characteristics and outcome of infective endocarditis involving implantable cardiac devices. JAMA. 2012;307:1727-1735.

25. Voigt A, Shalaby A, Saba S. Continued rise in rates of cardiovascular implantable electronic device infections in the United States: Temporal trends and causative insights. Pacing Clin Electrophysiol. 2010; 33:414-419. 
26. Baman TS, Gupta SK, Valle JA, et al. Risk factors for mortality in patients with cardiac device-related infection. Circ Arrhythm Electrophysiol. 2009:2:129-134.

27. Corman LC, Levison ME. Sustained bacteremia and transvenous cardiac pacemakers. JAMA. 1975;233:264-266.

28. Sohail MR, Henrikson CA, Braid-Forbes MJ, et al. Mortality and cost associated with cardiovascular implantable electronic device infections. Arch Intern Med. 2011;171:1821-1828.

29. Kempa M, Budrejko S, Piepiorka-Broniecka M, et al. One-year follow-up of patients undergoing transvenous extraction of pacemaker and defibrillator leads. PLoS One. 2015;10(12):e0144915.

30. Habib A, Le KY, Baddour LM, et al. Predictors of mortality in patients with cardiovascular implantable electronic device infections. Am J Cardiol. 2013;111:874-879.

31. Bongiorni MG, Tascini C, Tagliaferri E, et al. Microbiology of cardiac implantable electronic device infections. Europace. 2012;14:1334-1339.

32. Kratz JM, Toole JM. Pacemaker and internal cardioverter defibrillator lead extraction: A safe and effective surgical approach. Ann Thorac Surg. 2010;90:1411-1417.

33. Jan E, Camou F, Texier-Maugein J, et al. Microbiologic characteristics and in vitro susceptibility to antimicrobials in a large population of patients with cardiovascular implantable electronic device infection. J Cardiovasc Electrophysiol. 2012;23:375-381.

34. Rodriguez DJ, Afzal A, Evonich R, et al. The prevalence of methicillin resistant organisms among pacemaker and defibrillator implant recipients. Am J Cardiovasc Dis. 2012;2:116-122.

35. Le Dolley Y, Thuny F, Mancini J, et al. Diagnosis of cardiac device-related infective endocarditis after device removal. JACC CardiovasC Imaging. 2010;3:673-681.

36. Sohail MR, Uslan DZ, Khan AH, et al. Infective endocarditis complicating permanent pacemaker and implantable cardioverter-defibrillator infection. Mayo Clin Proc. 2008;83:46-53.

37. Ipek EG, Guray U, Demirkan B, et al. Infections of implantable cardiac rhythm devices: Predisposing factors and outcome. Acta Cardiol. 2012;67:303-310.

38. Rusanov A, Spotnitz HM. A 15-year experience with permanent pacemaker and defibrillator lead and patch extractions. Ann Thorac Surg 2010;89:44-50.

39. Massoure PL, Reuter S, Lafitte S, et al. Pacemaker endocarditis: Clinical features and management of 60 consecutive cases. Pacing Clin Electrophysiol. 2007;30:12-19.

40. Klug D, Lacroix D, Savoye C, et al. Systemic infection related to endocarditis on pacemaker leads: Clinical presentation and management. Circulation. 1997;95:2098-2107.

41. Cacoub P, Leprince $P$, Nataf $P$, et al. Pacemaker infective endocarditis. Am J Cardiol. 1998;82:480-484.
42. Viganego F, O'Donoghue S, Eldadah Z, et al. Effect of early diagnosis and treatment with percutaneous lead extraction on survival in patients with cardiac device infections. Am J Cardiol. 2012;109: 1466-1471.

43. Bensimhon L, Lavergne T, Hugonnet F, et al. Whole body [(18)F]fluorodeoxyglucose positron emission tomography imaging for the diagnosis of pacemaker or implantable cardioverter defibrillator infection: A preliminary prospective study. Clin Microbiol Infect. 2011;17: 836-844.

44. Lakkireddy D, Valasareddi S, Ryschon K, et al. The impact of povidone-iodine pocket irrigation use on pacemaker and defibrillator infections. Pacing Clin Electrophysiol. 2005;28:789-794.

45. del Rio A, Anguera I, Miro JM, et al. Surgical treatment of pacemaker and defibrillator lead endocarditis: The impact of electrode lead extraction on outcome. Chest. 2003;124:1451-1459.

46. Seifert $H$, Wisplinghoff $H$, Schnabel $P$, et al. Small colony variants of Staphylococcus aureus and pacemaker-related infection. Emerg Infect Dis. 2003;9:1316-1318.

47. Sohail MR, Uslan DZ, Khan AH, et al. Management and outcome of permanent pacemaker and implantable cardioverter-defibrillator infections. J Am Coll Cardiol. 2007;49:1851-1859.

48. Baddour LM, Epstein AE, Erickson CC, et al. Update on cardiovascular implantable electronic device infections and their management: A scientific statement from the American Heart Association. Circulation. 2010;121:458-477.

49. McCann P. A review of temporary cardiac pacing wires. Indian Pacing Electrophysiol J. 2007;7:40-49.

50. Darouiche R, Mosier M, Voigt J. Antibiotics and antiseptics to prevent infection in cardiac rhythm management device implantation surgery. Pacing Clin Electrophysiol. 2012;35:1348-1360.

51. Da Costa A, Kirkorian G, Cucherat M, et al. Antibiotic prophylaxis for permanent pacemaker implantation: A meta-analysis. Circulation. 1998;97:1796-1801.

52. Bluhm G, Nordlander R, Ransjo U. Antibiotic prophylaxis in pacemaker surgery: A prospective double blind trial with systemic administration of antibiotic versus placebo at implantation of cardiac pacemakers. Pacing Clin Electrophysiol. 1986;9:720-726.

53. Bratzler DW, Dellinger EP, Olsen KM, et al. Clinical practice guidelines for antimicrobial prophylaxis in surgery. Am J Health Syst Pharm. 2013; 70:195-283.

54. Lee I, Agarwal RK, Lee BY, et al. Systematic review and cost analysis comparing use of chlorhexidine with use of iodine for preoperative skin antisepsis to prevent surgical site infection. Infect Control Hosp Epidemiol. 2010;31:1219-1229.

55. Darouiche RO, Wall MJ, Itani KMF, et al. Chlorhexidine-alcohol versus povidone-iodine for surgical-site antisepsis. NEngl J Med. 2010;362: 18-26. 